\title{
Near-Field THz Nanoscopy with Novel Accelerator-Based Photon Sources ${ }^{\dagger}$
}

\author{
Lukas M. Eng 1,2,* , Frederik Kuschewski ${ }^{1}$, Jonathan Döring ${ }^{1}$, Lukas Wehmeier ${ }^{1}$, Tobias Nörenberg ${ }^{1}$, \\ Thales de Oliveira ${ }^{1}$, Hans-Georg von Ribbeck ${ }^{1}$, Denny Lang ${ }^{3}$, Bert Green ${ }^{3}$, Sergey Kovalev ${ }^{3}$, \\ Nilesh Awari ${ }^{3}$, Stephan Winnerl ${ }^{3}$, Manfred Helm ${ }^{3}$, Michael Gensch ${ }^{3}$ and Susanne C. Kehr ${ }^{1,2}$ \\ 1 Institute of Applied Physics, School of Science, TU Dresden, 01062 Dresden, Germany \\ 2 ct.qmat: Würzburg-Dresden Cluster of Excellence-EXC 2147, 01062 Dresden, Germany \\ 3 Helmholtz-Zentrum Dresden-Rossendorf, 01328 Dresden, Germany \\ * Correspondence: lukas.eng@tu-dresden.de \\ † Presented at the 37th International Symposium on Dynamical Properties of Solids (DyProSo 2019), Ferrara, \\ Italy, 8-12 September 2019.
}

Published: 5 September 2019

This talk advertises scattering-type scanning near-field infrared/THz nanospectroscopy (sSNIM) in the spectral range of 75 to $1.2 \mathrm{THz}[1,2]$, as provided by the free-electron laser FELBE at the Helmholtz-Zentrum Dresden-Rossendorf (HZDR), Germany. By combining s-SNIM with FELBE, we demonstrate a $\lambda$-independent optical resolution of $\sim 10 \mathrm{~nm}$ only, by exploring structured Au samples, Graphene-nanotransistors, meta-materials [3,4], and local-scale ferroic phase-transitions [5-7] down to LHe temperatures [8]. Moreover, also the non-linear optical responses at IR wavelengths can be explored, as recently demonstrated when inspecting highly-doped GaAs/InGaAs core/shell nanowires [9]. Our THz-s-SNIM was also integrated into a THz pump-probe setup for the local analysis of excited states in structured SiGe samples. We developed a sophisticated demodulation technique that extracts pump-induced signals with a superior signal-to-noise ratio [10]. In addition, HZDR recently extended the available wavelength ranges down to the $100 \mathrm{GHz}$ radiation, employing the novel super-radiant TELBE light source [11,12]. We adapted our s-SNIM to that novel TELBE photon source as well, achieving an equally high spatial resolution as with FELBE. This now allows to bridge the famous THz-gap in order to explore novel quantum phenomena of magnons, spin waves, and phonon polaritons in a various 2D and 3D materials.

\section{References}

1. Kuschewski, F.; von Ribbeck, H.-G.; Döring, J.; Winnerl, S.; Eng, L.M.; Kehr, S.C. Narrow-band near-field nanoscopy in the spectral range from 1.3 to $8.5 \mathrm{THz}$. Appl. Phys. Lett. 2016, 108, 113102.

2. Kehr, S.C.; Döring, J.; Gensch, M.; Helm, M.; Eng, L.M. FEL-based near-field infrared to THz nanoscopy. Synch. Rad. News 2017, 30, 31.

3. Kehr, S.C.; McQuaid R.G.P.; Kämpfe, T.; Kuschewski, F.; Lang, D.; Döring, J.; Gregg, J.M.; Eng, L.M. A local Superlens. ACS Photonics 2016, 3, 20.

4. Fehrenbacher, M.; Winnerl, S.; Schneider, H.; Döring, J.; Kehr, S.C.; Eng, L.M.; Huo, Y.; Schmidt, O.G.; Yao, K.; Liu, Y.; et al. Plasmonic Superlensing in Doped GaAs. Nano Lett. 2015, 15, 1057.

5. Döring, J.; Eng, L.M.; Kehr, S. Low-temperature piezoresponse force microscopy on barium titanate. J. Appl. Phys. 2016, 120, 084103.

6. Döring, J.; von Ribbeck, H.-G.; Fehrenbacher, M.; Kehr, S.C.; Eng, L.M. Near-field resonance shifts of ferroelectric barium titanate domains upon low-temperature phase transition. Appl. Phys. Lett. 2014, 105, 053109 .

7. Butykai, A.; Bordacs, S.; Kezsmarki, I.; Tsurkan, V.; Loidl, A.; Döring, J.; Neuber, E.; Milde, P.; Kehr, S.C.; Eng, L.M. Characteristics of ferroelectric-ferroelastic domains in Néel-type skyrmion host GaV4Ss. Sci. Rep. $2017,7,44663$. 
8. Lang, D.; Döring, J.; Nörenberg, T.; Butykai, A.; Kézsmàrki, I.; Schneider, H.; Winnerl, S.; Helm, M.; Kehr, S.C.; Eng, L.M. Infrared nanoscopy down to liquid helium temperatures. Rev. Sci. Instrum. 2018, 89, 033702.

9. Lang, D.; Balaghi, L.; Winnerl, S.; Schneider, H.; Hübner, R.; Kehr, S.; Eng, L.M.; Helm, M.; Dimakis, E.; Pashkin, A. Nonlinear plasmonic response of doped nanowires observed by infrared nanospectroscopy. Nanotechnology 2019, 30, 084003.

10. Kuschewski, F.; Kehr, S.C.; Green, B.; Bauer, Ch.; Gensch, M.; Eng, L.M. Optical nanoscopy of transient states in condensed matter. Sci. Rep. 2015, 5, 12582.

11. Green, B.; Kovalev, S.; Asgekar, V.; Geloni, G.; Lehnert, U.; Golz, T.; Kuntzsch, M.; Bauer, C.; Hauser, J.; Voigtlaender, J.; et al. High-Field High-Repetition-Rate Sources for the Coherent THz Control of Matter. Sci. Rep. 2016, 6, 22256.

12. Kovalev, S.; Green, B.; Golz, T.; Maehrlein, S.; Stojanovic, N.; Fisher, A.S.; Kampfrath, T.; Gensch, M. Probing ultra-fast processes with high dynamic range at 4th-generation light sources: Arrival time and intensity binning at unprecedented repetition rates. Struct. Dyn. 2017, 4, 024301.

(C) 2018 by the authors. Licensee MDPI, Basel, Switzerland. This article is an open access article distributed under the terms and conditions of the Creative Commons Attribution (CC BY) license (http://creativecommons.org/licenses/by/4.0/). 